\title{
Controlling and reporting for strategy
}

\author{
Luciano Marchi ${ }^{*}$, Giulio Greco**
}

Management Control is an academic journal devoted to the advancement of management control, management accounting and information systems knowledge. The journal provides a forum for the exchange of insights, knowledge and information based on both theoretical development and empirical evidence.

Our mission to publish high quality research manuscripts goes on with this special issue of Management Control, including a selection of five papers presented at the $1^{\text {st }}$ Workshop of the Management Control Journal "Controlling and reporting for strategy", held in Pisa in February 2012.

Drawing on the literature on accounting change and on the impacts of organizational life-cycle on management accounting systems, the first paper by Zoni, Dossi and Morelli discusses how the MAS change process differentiates through the organization lifecycle. The field study suggests that, to achieve successful MAS changes, the organization should focus on the design phase at the organizations' birth and at its revival stages. In the growth, maturity and decline stages, the organization should instead focus on the implementation phase.

The second article by Chiucchi, Gatti and Marasca investigates the relationship between MAS and ERP systems in a medium-sized firm. In a departure from prior research, the Authors also focus on the way MAS can influence the design, the implementation and the use of ERP systems. Interestingly, the paper found evidence of a bidirectional relationship between MAS and ERP systems.

\footnotetext{
${ }^{*}$ Editor-In-Chief.

** Special Issue Assistant Editor.
} 
The third paper by Oliviero and Castellano provides an original case study on the information systems design for reporting and strategic management purposes. Drawing on the new institutional sociology theory, the paper analyses the selection and integration of information systems, aimed at creating an effective reporting process. In the sample firm, the CFO decided to re-design the architecture of existing information systems, by selecting the most suitable information tools, rather than implementing a standardized software package. The findings are of interest to both researchers and practitioners.

The fourth article by Cardoni explores the business planning and management accounting processes in strategic networks. The topic is of particular interest, given the possibilities to formalize the network "agreement", allowed by the Italian Law. The study provides a comparative case study on fifty network "agreements", highlighting the relevant theoretical and practical implications of an emerging phenomenon in its early stages.

The fifth paper by Silvi, Bartolini, Raffoni and Vissani studies the Business Performance Analytics (BPA) as a framework for PMS design and adoption. BPA is defined as the use of data and analytical methods to understand and control business dynamics and performance. Through a survey, the Authors find evidence that companies implementing business intelligence and advanced PMS tools have a more clear perception about the usefulness of statistical and mathematical tools to support the decision-making process.

I encourage you to read in detail those articles which interest you most, as they have the potential to contribute to prior academic literature on management control, management accounting and information systems. 